\title{
Best Lower and Upper Approximates to Irrational Numbers
}

\author{
Clark Kimberling \\ Clark Kimberling received his Ph.D. in 1970 from the Illinois Institute of Technology. \\ Aside from number theory, his interests include triangle geometry, playing musical \\ instruments, and composing.
}

Wherever fractional notation of the form $p / q$ occurs in this paper, $p$ and $q$ denote relatively prime positive integers. The rational number $p / q$ is a best approximate to $\alpha$ (e.g., Lang [L]) if for every $b / c$ having denominator $c<q$,

$$
|q \alpha-p|<|c \alpha-b|
$$

These conditions imply $q|\alpha-p / q|<c|\alpha-b / c|<q|\alpha-b / c|$, so that

$$
|\alpha-p / q|<|\alpha-b / c|
$$

which is to say that $p / q$ is nearer $\alpha$ than any $b / c$ having $c<q$. However, (1) is stronger than (2), as exemplified by $(\alpha, p / q, b / c)=(1,3 / 5,1 / 2)$; in other words "best approximate" is "better" than "nearest approximate".

Given a positive irrational number $\alpha$, the principal convergents $p_{i} / q_{i}$ to $\alpha$ are well known to be the best approximates to $\alpha$; this theorem lends itself to a lemma proved in [L]:

Lemma 1 Suppose $p_{0} / q_{0}, p_{1} / q_{1}, \ldots$ are the principal convergents to a positive irrational number $\alpha$. If $b / c$ satisfies $|c \alpha-b|<\left|q_{i} \alpha-p_{i}\right|$, then $c \geq q_{i+1}$.

Die Approximation irrationaler Zahlen durch rationale Zahlen spielt in der Zahlentheorie eine grosse Rolle. Erinnert sei zum Beispiel an den berühmten Satz von Liouville; er liefert die Möglichkeit, die Transzendenz einer reellen Zahl mit Hilfe ihrer rationalen Approximationen zu beweisen. Clark Kimberling konstruiert im vorliegenden Beitrag auf einfache Weise beste untere und obere rationale Approximationen und setzt sie in Beziehung zu den bereits von Perron eingeführten "besten" und "nächsten Näherungen". ust 
We modify inequality (1), calling $p / q$ a best lower approximate to $\alpha$ if $p / q<\alpha$ and for every $b / c<\alpha$ having $c<q$,

$$
q \alpha-p<c \alpha-b
$$

and calling $p / q$ a best upper approximate to $\alpha$ if $p / q>\alpha$ and for every $b / c<\alpha$ having $c<q$

$$
p-q \alpha<b-c \alpha \text {. }
$$

Before solving for $p / q$ in these cases, we note that the analogous problem for "nearest" lower and upper approximates is solved in Perron [P, pp. 55-63], where, ironically, they are called "beste Näherungen". Perron's solutions are the same as those obtained below - a surprise in view of the aforementioned nonequivalence of "nearest" and "best".

For irrational $x$, let $\|x\|$ denote the distance from $x$ to the integer nearest to $x$. Let $\lfloor x\rfloor$ denote the greatest integer $\leq x$, and define $((x))$, the fractional part of $x$, by $((x))=x-\lfloor x\rfloor$. Then

$$
\|x\|= \begin{cases}((x)) & \text { if }((x))<1 / 2 \\ 1-((x)) & \text { otherwise }\end{cases}
$$

and (1) can be written as $\|q \alpha\|<\|c \alpha\|$.

Next we recall some basics about continued fractions, principal convergents, and intermediate convergents. Suppose $\alpha$ has continued fraction $\llbracket a_{0}, a_{1}, a_{2}, \ldots \rrbracket$, and let

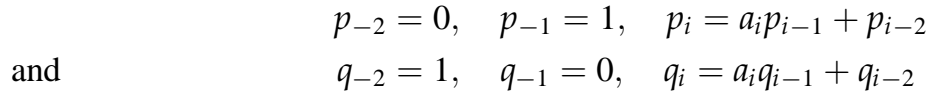

for $i \geq 0$. The principal convergents of $\alpha$ are the rational numbers $p_{i} / q_{i}$ for $i \geq 0$.

Now for all nonnegative integers $i$ and $j$, define

$$
p_{i, j}=j p_{i+1}+p_{i} \text { and } q_{i, j}=j q_{i+1}+q_{i}
$$

The fractions

$$
\frac{p_{i, j}}{q_{i, j}}=\frac{j p_{i+1}+p_{i}}{j q_{i+1}+q_{i}}, \quad 1 \leq j \leq a_{i+2}-1,
$$

are the $i$-th intermediate convergents of $\alpha$. As proved in [L, p. 16],

$$
\begin{array}{ll}
\cdots<\frac{p_{i}}{q_{i}}<\cdots<\frac{p_{i, j}}{q_{i, j}}<\frac{p_{i, j+1}}{q_{i, j+1}}<\cdots<\frac{p_{i+2}}{q_{i+2}}<\cdots & \text { if } i \text { is even, } \\
\cdots>\frac{p_{i}}{q_{i}}>\cdots>\frac{p_{i, j}}{q_{i, j}}>\frac{p_{i, j+1}}{q_{i, j+1}}>\cdots>\frac{p_{i+2}}{q_{i+2}}>\cdots \quad \text { if } i \text { is odd, }
\end{array}
$$

and $p_{i, j-1} q_{i j}-p_{i j} q_{i, j-1}=(-1)^{j}$ for $i=0,1,2, \ldots$ and $j=1,2, \ldots, a_{i+2}-1$. If the range of $j$ in (4) is extended to $0 \leq j \leq a_{i+2}-1$, then the principal convergents are included among the intermediate convergents. We shall refer to both kinds as simply convergents, those in (5) as even-indexed convergents, and those in (6) as odd-indexed convergents.

Suppose now that $q \geq 1$. Taking $x=q \alpha$ in (3) gives $\|q \alpha\|=|q \alpha-p|$, where

$$
p=\left\{\begin{array}{ll}
\lfloor q \alpha\rfloor & \text { if }((q \alpha))<1 / 2 \\
\lfloor q \alpha\rfloor+1 & \text { otherwise, }
\end{array} \text { so that }\|q \alpha\|= \begin{cases}((q \alpha)) & \text { if }((q \alpha))<1 / 2 \\
1-((q \alpha)) & \text { otherwise. }\end{cases}\right.
$$


Lemma 2 If $p_{i} / q_{i}$ are the principal convergents to a positive irrational number $\alpha$ and $i$ is even, then $\left(\left(q_{i+2} \alpha\right)\right)<1 / 2<\left(\left(q_{i+1} \alpha\right)\right)$,

$$
\left(\left(j q_{i+1} \alpha\right)\right)=j\left(\left(q_{i+1} \alpha\right)\right)-j+1
$$

and

$$
\left(\left(j q_{i+1} \alpha\right)\right)+\left(\left(q_{i} \alpha\right)\right)>1
$$

for $j=1,2, \ldots, a_{i+2}-1$.

Proof. The first assertion merely expresses the fact that the integer nearest $q_{i} \alpha$ is $p_{i}$ for even $i>0$, and that the integer nearest $q_{i+1} \alpha$ is $p_{i}+1$. Continuing, it is well known ([L, p. 8]) that

$$
p_{i+1}-q_{i+1} \alpha<1 / q_{i+2}
$$

so that $\left(\left(q_{i+1} \alpha\right)\right)>1-1 / q_{i+2}$. Now $a_{i+2} \leq a_{i+2} q_{i+1}+q_{i}=q_{i+2}$, whence

$$
0<j\left(\left(q_{i+1} \alpha\right)\right)-j+1<1 \text { for } 1 \leq j \leq a_{i+2}-1 .
$$

Since $\left(\left(j q_{i+1} \alpha\right)\right)$ is an irrational number having the same fractional part as $j\left(\left(q_{i+1} \alpha\right)\right)$ $j+1$, identity (7) is proved. Continuing,

$$
p_{i+2} q_{i+1}-q_{i+2} p_{i+1}=-1<0=p_{i+2} q_{i+2}-q_{i+2} p_{i+2}
$$

so that

$$
\alpha>\frac{p_{i+2}}{q_{i+2}}>\frac{p_{i+2}-p_{i+1}}{q_{i+2}-q_{i+1}} .
$$

Then $\alpha\left(a_{i+2} q_{i+1}+q_{i}-q_{i+1}\right)>a_{i+2} p_{i+1}+p_{i}-p_{i+1}$, which implies

$$
\frac{\left(\left(q_{i} \alpha\right)\right)}{1-\left(\left(q_{i+1} \alpha\right)\right)}=\frac{q_{i} \alpha-p_{i}}{p_{i+1}-q_{i+1} \alpha}>a_{i+2}-1 .
$$

Thus for $0 \leq j \leq a_{i+2}-1$, we have

$$
j\left(1-\left(\left(q_{i+1} \alpha\right)\right)\right)<\left(\left(q_{i} \alpha\right)\right)
$$

so that

$$
j\left(\left(q_{i+1} \alpha\right)\right)-j+1+\left(\left(q_{i} \alpha\right)\right)>1
$$

and (8) follows from (7).

Lemma 3 If $p_{i j} / q_{i j}$ are the convergents to a positive irrational number $\alpha$ and $i$ is even, then

$$
\left(\left(q_{i 0} \alpha\right)\right)>\left(\left(q_{i 1} \alpha\right)\right)>\left(\left(q_{i 2} \alpha\right)\right)>\cdots>\left(\left(q_{i, a_{i+2}-1} \alpha\right)\right)>1-\left(\left(q_{i+1,0} \alpha\right)\right)>\left(\left(q_{i+2,0} \alpha\right)\right) .
$$


Proof. When $j=0$, we are dealing with principal convergents, hence best approximates to $\alpha$, so that

or equivalently,

$$
\left\|q_{i 0} \alpha\right\|>\left\|q_{i+1,0} \alpha\right\|>\left\|q_{i+2,0} \alpha\right\|
$$

$$
\left(\left(q_{i 0} \alpha\right)\right)>1-\left(\left(q_{i+1,0} \alpha\right)\right)>\left(\left(q_{i+2,0} \alpha\right)\right) .
$$

Next, using Lemma 2 , we find for $j=0,1, \ldots, a_{i+2}-2$ that

$$
\left(\left(j q_{i+1} \alpha\right)\right)=j\left(\left(q_{i+1} \alpha\right)\right)-j+1>(j+1)\left(\left(q_{i+1} \alpha\right)\right)-(j+1)+1=\left(\left((j+1) q_{i+1} \alpha\right)\right),
$$

so that $\left(\left(q_{i j} \alpha\right)\right)>\left(\left(q_{i, j+1} \alpha\right)\right)$. Finally,

$$
1-\left(\left(q_{i+1} \alpha\right)\right)=p_{i+1}-q_{i+1} \alpha<1 / q_{i+2}<1 / a_{i+2},
$$

whence $a_{i+2}\left(\left(q_{i+1} \alpha\right)\right)>a_{i+2}-1$, and

$$
\left(\left(q_{i, a_{i+2}-1} \alpha\right)\right)=\left(a_{i+2}-1\right)\left(\left(q_{i+1} \alpha\right)\right)-\left(a_{i+2}-1\right)+1>1-\left(\left(q_{i+1} \alpha\right)\right) .
$$

Theorem 1 The best lower approximates to a positive irrational number $\alpha$ are the even-indexed convergents to $\alpha$.

Proof. Suppose $q_{i j}$ is an even-indexed convergent, and $c$ is a positive integer such that $((c \alpha))<\left(\left(q_{i j} \alpha\right)\right)$. We wish to show that $c>q_{i j}$. By Lemma 3, $((c \alpha))<\left(\left(q_{i} \alpha\right)\right)$, so that $\|c \alpha\|<\left\|q_{i} \alpha\right\|$, which by Lemma 1 implies $c \geq q_{i+1}$. If $c=q_{i+1}$, then $((c \alpha))=\left(\left(q_{i+1} \alpha\right)\right)$, which by Lemma 3 implies

$$
((c \alpha))>1-\left(\left(q_{i j} \alpha\right)\right)>\left(\left(q_{i j} \alpha\right)\right),
$$

a contradiction. Also, clearly, $c \neq q_{i j}$, so it remains to consider the possibility that $q_{i+1}<c<q_{i j}$; write $c=m q_{i+1}+h$, where $1 \leq h<q_{i+1}$ and $1 \leq m \leq j$. Then

$$
((c \alpha))=\left(\left(m q_{i+1} \alpha+h \alpha\right)\right)
$$

and, using Lemma 2,

$\left(\left(m q_{i+1} \alpha\right)\right)+((h \alpha))-1=((c \alpha))<\left(\left(q_{i j} \alpha\right)\right)=\left(\left(j q_{i+1} \alpha+q_{i} \alpha\right)\right)=\left(\left(j q_{i+1} \alpha\right)\right)+\left(\left(q_{i} \alpha\right)\right)-1$,

so that

$$
((h \alpha))<\left(\left(j q_{i+1} \alpha\right)\right)-\left(\left(m q_{i+1} \alpha\right)\right)+\left(\left(q_{i} \alpha\right)\right) .
$$

Identity (7) easily gives $\left(\left(j q_{i+1} \alpha\right)\right) \leq\left(\left(m q_{i+1} \alpha\right)\right)$, so that $((h \alpha))<\left(\left(q_{i} \alpha\right)\right)$, and by Lemma $1, h>q_{i+1}$, a contradiction.

Lemma 4 Let $p_{i} / q_{i}$ denote the principal convergents to a positive irrational number $\alpha=\llbracket a_{0}, a_{1}, a_{2}, \ldots \rrbracket$, and let $p_{i}^{\prime} / q_{i}^{\prime}$ denote the principal convergents to the number $\alpha^{\prime}=$ $a_{0}+1-\alpha$. If $\alpha-a_{0}<1 / 2$, then $p_{0}^{\prime} / q_{0}^{\prime}=0 / 1, p_{1}^{\prime} / q_{1}^{\prime}=1 / 1$, and

$$
\frac{p_{i}^{\prime}}{q_{i}^{\prime}}=\frac{\left(a_{0}+1\right) q_{i-1}-p_{i-1}}{q_{i-1}}
$$

for $i=2,3, \ldots$. If $\alpha-a_{0}>1 / 2$, then $a_{1}=1, p_{0}^{\prime} / q_{0}^{\prime}=0 / 1, p_{1}^{\prime} / q_{1}^{\prime}=1 /\left(a_{2}+1\right)$, and

for $i=2,3, \ldots$.

$$
\frac{p_{i}^{\prime}}{q_{i}^{\prime}}=\frac{\left(a_{0}+1\right) q_{i+1}-p_{i+1}}{q_{i+1}}
$$


Proof. It is easy to verify that

$$
\alpha^{\prime}= \begin{cases}\llbracket 0,1, a_{1}-1, a_{2}, a_{3}, a_{4}, \ldots \rrbracket & \text { if } \alpha-a_{0}<1 / 2 \\ \llbracket 0, a_{2}+1, a_{3}, a_{4}, \ldots \rrbracket & \text { if } \alpha-a_{0}>1 / 2 .\end{cases}
$$

The rest of the proof is routine and omitted.

Theorem 2 The best upper approximates to a positive irrational number $\alpha$ are the odd-indexed convergents to $\alpha$.

Proof. Suppose $p_{i j} / q_{i j}$ is an odd-indexed convergent to $\alpha$. Suppose also, contrary to (1U), that there exists $b / c$, with denominator $c<q_{i j}$, such that

$$
p_{i j}-q_{i j} \alpha \geq b-c \alpha .
$$

Substituting $a_{0}+1-\alpha^{\prime}$ for $\alpha$ and using principal convergents, we then have

$$
\begin{aligned}
& \left(j p_{i+1}+p_{i}\right)-\left(j q_{i+1}+q_{i}\right)\left(a_{0}+1-\alpha^{\prime}\right) \geq b-c\left(a_{0}+1-\alpha^{\prime}\right), \\
& \left(j q_{i+1}+q_{i}\right) \alpha^{\prime}-\left(j\left(\left(a_{0}+1\right) q_{i+1}-p_{i+1}\right)+\left(a_{0}+1\right) q_{i}-p_{i}\right) \geq c \alpha^{\prime}-\left(c a_{0}+c-b\right) .
\end{aligned}
$$

If $\alpha-a_{0}<1 / 2$, then by Lemma 4 , inequality (9) can be written as

$$
\left(j q_{i+2}^{\prime}+q_{i+1}^{\prime}\right) \alpha^{\prime}-\left(j p_{i+2}^{\prime}+p_{i+1}^{\prime}\right) \geq c \alpha^{\prime}-\left(c a_{0}+c-b\right),
$$

so that

$$
q_{i+1, j}^{\prime} \alpha^{\prime}-p_{i+1, j}^{\prime} \geq c \alpha^{\prime}-\left(c a_{0}+c-b\right),
$$

contrary to Theorem 1 , since $p_{i+1, j}^{\prime} / q_{i+1, j}^{\prime}$ is an even-indexed convergent to $\alpha^{\prime}$ and

$$
c<q_{i j}=j q_{i+1, j}+q_{i}=j q_{i+2, j}^{\prime}+q_{i+1}^{\prime}=q_{i+1, j}^{\prime} .
$$

On the other hand, if $\alpha-a_{0}>1 / 2$, then (9) can be written as

$$
\left(j q_{i}^{\prime}+q_{i-1}^{\prime}\right) \alpha^{\prime}-\left(j p_{i}^{\prime}+p_{i-1}^{\prime}\right) \geq c \alpha^{\prime}-\left(c a_{0}+c-b\right),
$$

so that

$$
q_{i-1, j}^{\prime} \alpha^{\prime}-p_{i-1, j}^{\prime} \geq c \alpha^{\prime}-\left(c a_{0}+c-b\right),
$$

contrary to Theorem 1 .

\section{References}

[L] Serge Lang, Introduction to Diophantine Approximations, Addison-Wesley, Reading, Mass., 1966.

[P] Oskar Perron, Die Lehre von den Kettenbrüchen, Chelsea, New York, 1950.

Clark Kimberling

Department of Mathematics

University of Evansville

1800 Lincoln Avenue

Evansville, Indiana 47722 